\title{
Fibrolamellar hepatocellular carcinoma-related hyper- ammonemic encephalopathy: Up to now and next steps
}

\author{
Rodrigo Cañada Trofo Surjan ${ }^{1,2}$, Elizabeth Santana dos Santos ${ }^{3}$, Sergio do Prado Silveira ${ }^{1,3}$, Fabio Ferrari Makdissi , \\ and Marcel Autran Cesar Machado²
}

'Department of Surgery, Hospital Nove de Julho, São Paulo; ${ }^{2}$ Department of Surgery, University of São Paulo Medical School, São Paulo; ${ }^{3}$ Department of Oncology, A.C. Camargo Cancer Center, São Paulo; ${ }^{4}$ Department of Gastrointestinal Surgery, University of São Paulo Medical School, São Paulo, Brazil

Dear Editor,

We recently had the opportunity ro read the brilliant paper by Thakral and Simonetto "Hyperammonemic encephalopathy: An unusual presentation of fibrolamellar hepatocellular carcinoma" in the Clinical and Molecular Hepatology.' We would appreciate to share some insights about this interesting theme.

Fibrolamellar hepatocellular carcinoma (FLHCC) is indeed a rare primary hepatic tumor that arises in non-cirrhotic livers, more common in males (male to female ratio 1.7), with age-specific incidence with two peaks between ages of 10-30 and 60-69 (although being a tumor usually believed to affect mostly very young individuals) and is associated with better 5 -year survival than conventional hepatocellular carcinoma (HCC). ${ }^{2}$ Although FLHCC was usually referred as a subtype of HCC, recent researches have demonstrated that FLHCC is actually an independent entity, with distinctive molecular tumor profile, histological features and clinical presentation. ${ }^{3,4}$

One of the most feared complications associated with FLHCC is the development of acute onset hyperammonemic encephalopathy (HAE), which was first reported by Sethi et al. ${ }^{5}$ in 2009, this condition is associated with high mortality. ${ }^{6}$

High mortality was related to the ignorance of the physiopa- thology of HAE in patients with FLHCC. Some authors have proposed some explanations, such as portosystemic shunt. ${ }^{5,7}$ In 2017, we published a new proposal of the physiopathology of this complication of FLHCC, and initiated with the very unique mutation (a heterozygous deletion of chromosome 19) that is responsible for the development of the tumor. This mutation is responsbile for overexperssion of a chimeric DNAJB1-PRKACA kinase and Aurora Kinase A that culminate with c-Myc and ornithine decarboxylase dysfunction and result in depletion of amino acids crucial to urea cycle function. ${ }^{8}$ This urea cycle dysfuntion is then responsible for the acummulation of ammonia in the bloodsteram and occurs HAE.

The importance of this finding was, once understanding that an urea cycle disorder due to metabolites consumption was responsible for the hyperammonemia, to guide the developement of new medical treatment options based on a combination of ammonia scavenger drugs (sodium benzoate and phenylbutyrate) with amino acids supplementation (citrulline, ornithine and arginine), thus reducing the mortality of FLHCC-related HAE and allowing complete clinical recovery from HAE. ${ }^{1,6,8}$ Table 1 summarizes all published reports on FLHCC-related HAE.

So, we think that question stated by Hashash et al. ${ }^{9}$ in 2012

\section{Abbreviations:}

FLHCC, fibrolamellar hepatocellular carcinoma; HCC, hepatocellular carcinoma; HAE, hyperammonemic encephalopathy

\section{Corresponding author : Rodrigo Cañada Trofo Surjan}

Department of Surgery, Hospital Nove de Julho, Rua Peixoto Gomide, 545. Cerqueira César, São Paulo 01409-002, Brazil

Tel: +5511 996519727, Fax: + 551121146077

E-mail: rodrigo.surjan@gmail.com

https://orcid.org/0000-0003-3918-8920 
Table 1. Published reports of fibrolamellar carcinoma related hyperammonemic encephalopathy

\begin{tabular}{|c|c|}
\hline Study & Place \\
\hline Sethi et al. ${ }^{5}$ (2009) & USA \\
\hline Berger et al.. (2012) & Argentina \\
\hline Hashash et al.' (2012) & USA \\
\hline Sulaiman and Geberhiwot ${ }^{10}$ (2014) & England \\
\hline Alsina et al."11 (2016) & USA \\
\hline Chapuy et al. ${ }^{12}$ (2016) & USA \\
\hline Surjan et al..$^{8}$ (2017) & Brazil \\
\hline Suarez et al. ${ }^{13}$ (2018) & Colombia \\
\hline Bartlett et al..$^{14}$ (2018) & USA \\
\hline Thakral and Simonetto ${ }^{1}$ (2019) & USA \\
\hline Cho et al. ${ }^{6}$ (2019) & USA \\
\hline
\end{tabular}

"What is the cause of acute hepatic encephalopathy in a young patient?" regarding an 18-year-old patient with a large FLHCC has been answered, including its precise pathophysiology. Nevetheless, a few more steps must still be taken. First, the molecular basis of the proposed pathophysiology is needed to be proven. Second, there were more studies are warranted to demosntrate the efficacy and safety of these new treatment options. Third, this treatment regimen for hyperammonia may be useful for other causes of liver-related HAE such as portosystemic shunt, acute liver failure and cirrhosis.

\section{Author's contributions}

Rodrigo Cañada trofo Surjan designed and wrote the paper, designed the proposal of the pathophysiology described and performed final approval of the article. Elizabeth Santana dos Santos was involved in direct patient care and data collection. Sergio do Prado Silveira performed data collection and paper drafting. Fabio Ferrari Makdissi and Marcel Autran Cesar Machado performed critical review of the article.

\section{Conflicts of Interest}

The authors have no conflicts to disclose.

\section{REFERENCES}

1. Thakral N, Simonetto DA. Hyperammonemic encephalopathy: an unusual presentation of fibrolamellar hepatocellular carcinoma. Clin Mol Hepatol 2020;26:74-77.

2. Eggert T, McGlynn KA, Duffy A, Manns MP, Greten TF, Altekruse SF. Epidemiology of fibrolamellar hepatocellular carcinoma in the USA, 2000-10. Gut 2013;62:1667-1668.

3. Fritz A, Percy C, Jack A, Shanmugaratnam K, Sobin L, Parkin DM, et al. International classification of diseases for oncology. 3. Geneva: World Health Organzation, 2010.

4. Lalazar G, Simon SM. Fibrolamellar carcinoma: recent advances and unresolved questions on the molecular mechanisms. Semin Liver Dis 2018;38:51-59.

5. Sethi S, Tageja N, Singh J, Arabi H, Dave M, Badheka A, et al. Hyperammonemic encephalopathy: a rare presentation of fibrolamellar hepatocellular carcinoma. Am J Med Sci 2009;338:522-524.

6. Cho J, Chen JCY, Paludo J, Conboy EE, Lanpher BC, Alberts SR, et al. Hyperammonemic encephalopathy in a patient with fibrolamellar hepatocellular carcinoma: case report and literature review. J Gastrointest Oncol 2019;10:582-588.

7. Berger $C$, Dimant $P$, Hermida L, Paulin F, Pereyra M, Tejo M. Encefalopatía hiperamoniémica y he- patocarcinoma fibrolamelar. Medicina (Buenos Aires) 2012;72:425-427.

8. Surjan RC, Dos Santos ES, Basseres T, Makdissi FF, Machado MA. a proposed physiopathological pathway to hyperammonemic encephalopathy in a non-cirrhotic patient with fibrolamellar hepatocellular carcinoma without ornithine transcarbamylase (OTC) mutation. Am J Case Rep 2017;18:234-241.

9. Hashash JG, Thudi K, Malik SM. An 18-year-old woman with a 15$\mathrm{cm}$ liver mass and an ammonia level of 342. Gastroenterology 2012;143:1157-1402.

10. Sulaiman RA, Geberhiwot T. Fibrolamellar hepatocellular carcinoma mimicking ornithine transcarbamylase deficiency. JIMD Rep 2014;16:47-50

11. Alsina AE, Franco E, Nakshabandi A, Albers C, Kemmer N, Berry AC, et al. Successful liver transplantation for hyperammonemic fibrolamellar hepatocellular carcinoma. ACG Case Rep J 2016;3:e106.

12. Chapuy Cl, Sahai I, Sharma R, Zhu AX, Kozyreva ON. Hyperammonemic encephalopathy associated with fibrolamellar hepatocellular carcinoma: case report, literature review, and proposed treatment algorithm. Oncologist 2016;21:514-520.

13. Suarez O, Perez M, Garzon M, Daza R, Hernandez G, Salinas C, et al. Fibrolamellar hepatocellular carcinoma and noncirrhotic hyperammonemic encephalopathy. Case Reports Hepatol 2018;2018:7521986.

14. Bartlett AL, Leslie ND, Gupta A, Geller JI. Acquired ornithine transcarbamylase deficiency in pediatric and adolescent patients with fibrolamellar hepatocellular carcinoma. Pediatr Blood Cancer 2018;65:e27392. 